\title{
Análisis perceptivo del tiempo por transcurrir y su impacto en el proceso productivo
}

\section{Perceptive analysis of time to pass and its impact on the production process}

\author{
Manuel Emilio Milla Pino' ${ }^{1}$, Danny Alberto Villegas Rivas ${ }^{2} \mathbb{D}$, Ricardo Ángel Shimabuku Ysa ${ }^{3}$, River Chávez Santos ${ }^{4} \mathbb{D}$
}

\section{RESUMEN}

El ser humano tiene distinta percepción del tiempo transcurrido y por transcurrir, aspecto que convierte al tiempo en elemento fundamental en la vida cotidiana y más aún en los procesos productivos, ya que las tareas inherentes a la producción requieren definir los tiempos de ejecución para poder sistematizarlas y planificarlas para lograr un secuenciamiento lógico y racional que garantice el éxito de las acciones emprendidas, de allí que analizar perceptivamente el tiempo por transcurrir es una valiosa acción a desarrollar.

Palabras claves: percepción, tiempo, transcurrir.

\begin{abstract}
The human being has a different perception of time elapsed and to elapse, an aspect that makes time a fundamental element in daily life and even more so in production processes, since the tasks inherent to production require defining the execution times in order to systematize them. and plan them to achieve a logical and rational sequencing that guarantees the success of the actions undertaken, hence the perceptual analysis of the time to elapse is a valuable action to be carried out.
\end{abstract}

Keywords: perception, time, elapse.

\footnotetext{
Universidad Nacional de Jaén, Facultad de Ingeniería Civil, Jaén, Perú

${ }^{2}$ Universidad Nacional de Jaén, Facultad de Ingeniería Forestal y Ambiental, Jaén, Perú

${ }^{3}$ Universidad Nacional de Jaén, Facultad de Ingeniería Mecánica y Eléctrica, Jaén, Perú

${ }^{4}$ Universidad Nacional Toribio Rodríguez de Mendoza, Facultad de Ciencias Económicas y Administrativas, Chachapoyas, Perú

*Autor de Correspondencia.E-mail: manuel.milla@unj.edu.pe
} 


\section{INTRODUCCIÓN}

Esta nota tiene como objetivo principal hacer un análisis de la percepción del tiempo, usando como base las impresiones que tienen los sujetos acerca del tiempo por transcurrir desde distintos escenarios, discutir la eficiencia del ser humano y discernir como la función productiva depende del tiempo. En este contexto se plantean diversas situaciones de la vida cotidiana donde el tiempo y su percepción asumen un rol fundamental para formular una visión de futuro.

\section{I . T I E M P O D E E S P ERA E N UN RESTAURANTE}

En el común de los casos, al llegar a un restaurante donde se ofrecen platos a la carta, el mesero luego de tomar el pedido, informa el tiempo aproximado en que la comida será servida. Asumamos que el tiempo estimado de espera es de 20 minutos, este lapso será un momento agradable y de utilidad para el abordaje de temas relevantes, informativos o de negocios, si los comensales no tienen un nivel de hambre exagerado, ya que si fuese lo contrario, ese lapso se convertiría en angustia y desespero, que haría de esta velada un espacio intolerante e inapropiado que puede traer como consecuencia que el momento no sea grato. En este orden de ideas se puede decir que el disfrute del tiempo de espera en un restaurant está vinculado al nivel de hambre que tenga los comensales (Hermida et al., 2004).

\section{III. ÚLTIMOS MINUTOS DE UN PARTIDO DE FÚTBOL}

En la mayoría de los partidos de fútbol, los árbitros o jueces decretan un tiempo de alargue o prórroga, y este tiempo es agradable para el equipo que está ganando, si y solo si la ventaja es marcada, ya que si la diferencia es mínima ese tiempo hace que el partido se haga interminable. En lo que respecta al equipo que está perdiendo, ese tiempo se percibe como muy corto e insuficiente para lograr empatar el partido y mucho más para ganarlo, lo cual evidencia que el juego termine muy rápido. Esto indica que los últimos minutos de un juego de fútbol serán interminables o insuficientes dependiendo de cómo vaya el score (Motta, 2004).

\section{TRASLADO ENTRE LOCALIDADES}

Aun cuando el tiempo que dura el traslado de una localidad a otra sea el mismo, ya que la distancia entre localidades es la misma, la percepción del tiempo de duración del viaje, dependerá de la premura o urgencia que se tenga en llegar a la localidad, esto indica que, si el viaje es con fines turísticos quizás el tiempo no sea un factor relevante, pero si el traslado es con fines médicos, cada minuto cuenta, lo que se traduce en una percepción del tiempo como elemento prioritario del viaje. En este sentido trasladarse de una localidad a otra generará una interpretación o percepción del tiempo, dependiendo del propósito del viaje (Krajewski et al., 2013; Chase et al., 2014).

\section{LA VIDA CADA DÍASE ACERCAAL FINAL}

La experiencia, competencias, habilidades y destrezas adquiridas formal e informalmente con el pasar del tiempo, se convierten en la carta de presentación del individuo ante el mercado laboral. Pero cuanto más ha tardado el individuo en obtener esas capacidades, menor es el tiempo de vida útil que le queda para hacer uso con eficacia y eficiencia de sus competencias. Este escenario permite aseverar que la adquisición de experiencia, utiliza un tiempo que al final se debe restar de la vida útil que tenemos los seres humanos, ya que la vida cada día se acerca al final (Zimmermaan, 2017).

\section{DISMINUCIÓN DE LA EFICIENCIA HUMANA}

La adquisición de conocimientos hace que el ser humano piense que sus condiciones físicas y mentales permanecen intactas con el pasar del tiempo, y esto lo lleva a expresar que las actividades que realizaba años atrás en cierto margen de tiempo en la actualidad también podrá efectuarlas en ese tiempo. Aunque no es fácil aceptarlo, resulta que en la medida que va transcurriendo el tiempo en esa misma medida la eficiencia del ser humano va disminuyendo, aspecto que condu- 
ce a que algunas personas señalen en forma coloquial que 'el año pasa volando' (Krajewski et al., 2000).

VII. RE FLEXIÓN ACERCA DE LA PRODUCCIÓN EN FUNCIÓN DELTIEMPO

Lo expuesto en los numerales anteriores, constituyen elementos que conducen a expresar la afirmación, de que en todo proceso productivo el tiempo es un factor fundamental y que la obtención de mayores o menores niveles de rendimientos está ligado a ese factor, lo que se traduce en la necesaria selección oportuna del momento de siembra, de los momentos de aplicación de productos orgánicos para el control de plagas y/o enfermedades, el momento apropiado para la cosecha y el tiempo de manejo del periodo de postcosecha, es decir, toda actividad inherente al proceso productivo tiene su momento y su duración.

\section{REFERENCIAS BIBLIOGRÁFICAS}

Krajewski, L. y L. P. Ritzman. 2000. Administración de operaciones: Estrategia y análisis. Nueva Jersey (EE.UU.): Pearson Prentice Hall

Krajewski, L., L. P. Ritzman, y M. K. Malhotra. 2013. Administración de operaciones: Procesos de cadenas de suministros. Nueva Jersey (EE.UU.): Pearson Prentice Hall

Chase, R. B., F. R. Jacobs, y N. J. Aquilano. 2014. Administración de operaciones: Producción y cadena de suministros. Ciudad de México (México): Mc Graw Hill.

Hermida, J. A., R. Serra, y E. Kastika. 2004. Administración y estrategia: teoría y práctica. Cali (Colombia): Grupo Editorial Norma

Motta, M. 2004. Competition policy: Theory andPractice. Cambridge (Reino Unido): Cambridge University Press.

Zimmermann, J. L. 2017). Accounting for decision making and control. Nueva York (EE.UU): Mc Graw Hill. 